\title{
Genetic Factors Associated with Susceptibility and Mortality in
} COVID-19

\author{
Fatemeh Bahraini (iD ${ }^{1}$, Forouzan Karam (iD) ${ }^{1}$ and Ebrahim Miri-Moghaddam (iD) ${ }^{2, *}$ \\ ${ }^{1}$ Student Research Committee, Department of Hematology and Blood Banking, School of Paramedical Sciences, Birjand University of Medical Sciences (BUMS), Birjand, Iran \\ ${ }^{2}$ Cardiovascular Disease Research Center, Department of Molecular Medicine, Faculty of Medicine, BUMS, Birjand, Iran \\ "Corresponding author: Cardiovascular Disease Research Center, Department of Molecular Medicine, Razi Hospital, BUMS, Birjand, Iran. Tel: +98-5631626461, Email: \\ moghaddam4@yahoo.com
}

Received 2021 July 04; Accepted 2021 July 26.

Keywords: COVID-19, Disease Severity, Epigenetics, Genetic Susceptibility, SARS-CoV-2

\section{Dear Editor,}

Clinical symptoms of COVID-19 are divided into four categories, according to studies and guidelines of the World Health Organization: mild, moderate, severe, and critical illness. Age, gender, and underlying diseases are factors that are associated with poor prognosis outcomes. Gender factor plays an important role in the severity of the consequences of COVID-19. Although the virus infection rate is similar between the two sexes, the mortality rate has been higher in males. Underlying diseases such as high blood pressure and diabetes have been seen in people with more severe and critical illnesses. However, these factors do not explain the severity of the disease in healthy young people, so there must be another factor related to the severity of the disease and susceptibility to the virus SARS-CoV2. Evidence from previous human viral infections suggests that the host genotype determines susceptibility or response to infection. In SARS-CoV-2, host genetics and genetic variants in specific gene clusters appear to be important in determining a person's susceptibility to the virus and the severity of COVID-19. Thus, the study of genetic and epigenetic factors associated with the virus and the severity of COVID-19 can improve our knowledge of the pathophysiology and pathogenicity of the virus. They could improve treatment methods and the production of individual vaccines based on genetics use in high-risk groups.

Among the well-known proteins are the angiotensinconverting enzyme (ACE) and Angiotensin-converting enzyme 2 (ACE2). ACE and ACE2 are genes that regulate the renin-angiotensin (RAS) system. Therefore, the balance between ACE/ACE2 is crucial for tuning the RAS system. The SARS-COV-2 enters the target cell using the receptorbinding domain (RBD) of the SARS-CoV-2 spike (S) protein to the ACE2 receptor at the target cell surface. Eleven protected amino acids were identified in ACE2 protein (T20, Y83, S218, A246, K353, P426, T593, N636, A714, R716, and A774) that were involved in facilitating virus entry with spike protein, and mutations in some of these block the virus from entering the cell (1). Therefore, the study of genetic variants of these genes improves the knowledge related to COVID-19. High levels of ACE in COVID-19 patients are harmful and cause tissue damage, fibrosis, thrombosis, and inflammation. There are polymorphisms of the ACE gene that have been linked to SAR-CoV2 virus susceptibility and mortality. Studies have shown the different frequency of alleles in the ACE gene in different populations. The p.Met383Thr and p.Asp427Tyr variants of ACE2 in the American African/African population and the p.Pro389His variant in the Latino/Admixed American population inhibit the interaction between the spike virus protein and ACE2. The expression level of the ACE2 gene varies in different people according to age, gender, and race. Important regulatory factors effectively express the ACE2 gene, including sex hormones (androgens), transcription factors associated with a viral infection, and epigenetic regulation. There is a hypothesis that androgen receptor signaling pathways are involved in regulating ACE2 and TMPRSS2 transcription. It may explain the difference in the severity of COVID-19 in the two sexes.

Epigenetic regulation of ACE2 is accomplished through methylation and acetylation of histones. DNA methylation in human lung tissue is highly variable in both men and women. Women are hypomethylated compared to men, so ACE2 gene expression is higher in women, and ACE2 has multiple physiological roles, protecting against organ damage, cardiovascular disease, and 
inflammation. Increased ACE2 expression is associated with a better prognosis because it reduces inflammation. Despite these findings, no significant association was found between ACE2 variants and the severity of COVID-19 in some studies (2).

The TMPRSS2 gene is located on chromosome 21 and encodes the protease enzyme serine, which cleaves and activates the $S$ virus protein during fusion into cells. Different gene variants have various expression levels and prevalence in different populations, leading to differences in COVID-19 susceptibility in different populations (3).

Among the genes on the $\mathrm{X}$ chromosome are PRRs (DDX3X, TLR7, TLR8) genes, TLR signaling regulators (IRAK1, NEMO), various immune-related genes (CD40L, CXCR3, CXorf21, FOXP3, IL3RA, TMEM187). One of the X chromosomes is accidentally inactivated to balance the expression of $\mathrm{X}$ genes between the sexes during random methylation. The escape of some genes from inactivation causes dual expression of the parents and hypersensitivity of the female immune system. The escape of TLR7 and TLR8 genes from inactivation causes more expression in women and ultimately increases antiviral function (4). The ACE2 gene is also located on the $\mathrm{X}$ chromosome and escapes inactivation, but due to the effect of sex hormones on the expression of this gene, it is difficult to investigate the actual effect of inactivation escape on ACE2 expression. Bivalent expression of ACE2 in women causes the formation of ACE2 heterodimer receptors that cannot bind to the RBD region of the S protein of the virus, whereas in men, these receptors are expressed as homodimers and lack ACE2 protective function in the organ (5).

In association with $\mathrm{ABO}$ loci with the severity of COVID19, blood group A has a higher risk, and blood group $O$ has a lower risk of COVID-19. ABO locus variants have different expression levels of CD209/DC-SIGN, the binding protein for SARS-COV2. It has been suggested that ABO loci pose a risk for COVID-19 and severe sequelae by altering DC-SIGN or affecting glycosyltransferase activity (6).

The genetic diversity of human leukocyte antigens (HLA) with sensitivity to the SARA-CoV-2 and the severity of COVID-19 was investigated. The presence of the HLA-B * 46: 01 allele increases susceptibility to COVID-19, and the HLA-B *15: 03 allele increases cellular immunity (7).

\section{Footnotes}

Authors' Contribution: Study concept and design, E. M.M., and F. B. and F.K.; Drafting of the manuscript, F. B.; F. K. and E.M.M; Critical revision of the manuscript for important intellectual content, E.M.M.

Conflict of Interests: The authors certify that they have NO affiliations with or involvement in any organization or entity with any financial interest (such as honoraria; educational grants; participation in speakers' bureaus; membership, employment, consultancies, stock ownership, or other equity interest; and expert testimony or patentlicensing arrangements), or non-financial interest (such as personal or professional relationships, affiliations, knowledge or beliefs) in the subject matter or materials discussed in this manuscript.

Funding/Support: No received.

\section{References}

1. Choudhary S, Sreenivasulu K, Mitra P, Misra S, Sharma P. Role of Genetic Variants and Gene Expression in the Susceptibility and Severity of COVID-19. Ann Lab Med. 2021;41(2):129-38. doi: 10.3343/alm.2021.41.2.129. [PubMed: 33063674]. [PubMed Central: PMC7591285].

2. Yildirim Z, Sahin OS, Yazar S, Bozok Cetintas V. Genetic and epigenetic factors associated with increased severity of Covid-19. Cell Biol Int. 2021;45(6):1158-74. doi: 10.1002/cbin.11572. [PubMed: 33590936]. [PubMed Central: PMC8014716].

3. Vargas-Alarcon G, Posadas-Sanchez R, Ramirez-Bello J. Variability in genes related to SARS-CoV-2 entry into host cells (ACE2, TMPRSS2, TMPRSS11A, ELANE, and CTSL) and its potential use in association studies. Life Sci. 2020;260:118313. doi: 10.1016/j.lfs.2020.118313. [PubMed: 32835700]. [PubMed Central: PMC7441892].

4. Anastassopoulou C, Gkizarioti Z, Patrinos GP, Tsakris A. Human genetic factors associated with susceptibility to SARS-CoV-2 infection and COVID-19 disease severity. Hum Genomics. 2020;14(1):40. doi: 10.1186/s40246-020-00290-4. [PubMed: 33092637]. [PubMed Central: PMC7578581].

5. Forsyth KS, Anguera MC. Time to get ill: the intersection of viral infections, sex, and the X chromosome. Curr Opin Physiol. 2021;19:62-72. doi: 10.1016/j.cophys.2020.09.015. [PubMed: 33073073]. [PubMed Central: PMC7553007].

6. Katz DH, Tahir UA, Ngo D, Benson M, Bick AG, Pampana A, et al. Proteomic Profiling in Biracial Cohorts Implicates DC-SIGN as a Mediator of Genetic Risk in COVID-19. medRxiv. 2020;Preprint. doi: 10.1101/2020.06.09.20125690.

7. Wang W, Zhang W, Zhang J, He J, Zhu F. Distribution of HLA allele frequencies in 82 Chinese individuals with coronavirus disease-2019 (COVID-19). HLA. 2020;96(2):194-6. doi: 10.1111/tan.13941. [PubMed: 32424945]. [PubMed Central: PMC7276866]. 